ANNALES

POLONICI MATHEMATICI

LXI.3 (1995)

\title{
Weak and strong topologies and integral equations in Banach spaces
}

\author{
by Donal O'Regan (Galway)
}

\begin{abstract}
The Schauder-Tikhonov theorem in locally convex topological spaces and an extension of Krasnosel'skiı̌'s fixed point theorem due to Nashed and Wong are used to establish existence of $L^{\alpha}$ and $C$ solutions to Volterra and Hammerstein integral equations in Banach spaces.
\end{abstract}

1. Introduction. This paper establishes existence of solutions to the Volterra integral equation

(1.1) $y(t)=h(t)+\int_{0}^{t} k(t, s) f(s, y(s)) d s \quad$ a.e. on $[0, T], \quad T>0$ is fixed,

and the Hammerstein integral equation

$$
y(t)=h(t)+\int_{0}^{1} k(t, s) f(s, y(s)) d s \quad \text { a.e. on }[0,1] .
$$

Here $y$ takes values in a real Banach space $B$.

In Section 2 existence of $L^{\alpha}([0, a], B)$ (with $\alpha>1, a=T$ or 1 ) solutions will be established for (1.1) and (1.2) where $B$ is a reflexive Banach space. In [6], C. Corduneanu first studied the Volterra equation in this setting. Our results extend and complement those in [6]. Also, our technique discusses naturally the interval of existence $[0, T]$. The method also extends so that we can examine the Hammerstein equation in the above setting. Throughout this section our analysis will rely on the Schauder-Tikhonov fixed point theorem in locally convex spaces.

Section 3 establishes existence of $C([0, a], B)$ solutions to (1.1) and (1.2); here $B$ will be a real Banach space. We will assume that $f$ has the splitting $f(t, u)=f_{1}(t, u)+f_{2}(t, u)$ where $f_{1}$ is a nonlinear contraction (to be

1991 Mathematics Subject Classification: 45D05, 45G10, 45N05.

Key words and phrases: Volterra, Hammerstein, existence, integral equations in abstract spaces. 
described later) on bounded sets and $f_{2}$ is completely continuous. The technique used will rely on an extension of Krasnosel'skiı's fixed point theorem [10] due to Nashed and Wong [16].

Some very interesting existence results for (1.1) and (1.2), in the case $B=\mathbb{R}$, may be found in $[3-5,13,14]$. For example, in [14] the Hammerstein equation (1.2), with $B=\mathbb{R}$, is examined and existence of $C[0,1]$ solutions is established if the nonlinearity $f$ satisfies a "sublinear" type growth condition. The Volterra equation (1.1), with $B=\mathbb{R}$, is discussed in [13]. Gripenberg, Londen and Staffans' basic idea is to show (1.1) has a (local) solution. They then discuss "continuation" of solutions. However, the interval of existence from a construction point of view is only briefly discussed.

For the remainder of this section we gather together some preliminaries that will be needed in Sections 2 and 3. Let $(\Omega, \Sigma, \mu)$ be a finite measure space. A Banach space $B$ has the Radon-Nikodym $(R-N)$ property with respect to $(\Omega, \Sigma, \mu)$ if for each $\mu$-continuous vector measure $\nu: \Sigma \rightarrow B$ of bounded variation there exists $g \in L^{1}(\mu, B)$ such that $\nu(E)=\int_{E} g d \mu$ for all $E \in \Sigma$.

TheOREM 1.1 [9]. If $B$ is a reflexive Banach space then $B$ has the $R-N$ property.

TheOrem $1.2[2]$. Let $(\Omega, \Sigma, \mu)$ be a finite measure space. Suppose $K \subseteq$ $L^{\alpha}(\mu, B), 1<\alpha<\infty$, is bounded with $K(A)=\left\{\int_{A} g d \mu: g \in K\right\}$ relatively weakly compact in $B$ for each $A \in \Sigma$. If $B$ and $B^{*}$ have the $R-N$ property then $K$ is relatively weakly compact.

TheOREm 1.3 [9]. Let $(\Omega, \Sigma, \mu)$ be a finite measure space, $1<\alpha<\infty$, and $B$ a Banach space. Then $\left(L^{\alpha}(\mu, B)\right)^{*}=L^{\beta}\left(\mu, B^{*}\right)$ where $1 / \alpha+1 / \beta=1$ iff $B^{*}$ has the $R-N$ property with respect to $\mu$.

Remark. In fact, for $\phi \in\left(L^{\alpha}(\mu, B)\right)^{*}$ there exists $g \in L^{\beta}\left(\mu, B^{*}\right)$ with

$$
\phi(f)=\int_{\Omega}\langle f, g\rangle d \mu \quad \text { for all } f \in L^{\alpha}(\mu, B) .
$$

Here $\langle f, g\rangle(t)=g(t)(f(t))$ for $t \in \Omega$.

TheOREM $1.4[7,11,17]$. A subset of a reflexive Banach space is weakly compact iff it is closed in the weak topology and bounded in the norm topology.

TheOREM $1.5[7,11,17]$. A convex subset of a normed space is closed iff it is weakly closed.

Theorem 1.6 (Schauder-Tikhonov) [3]. Let $K$ be a closed convex subset of a locally convex topological Hausdorff space $E$. Assume that $g: K \rightarrow K$ 
is continuous and that $g(K)$ is relatively compact in $E$. Then $g$ has at least one fixed point in $K$.

Theorem 1.7 [17]. Let $B_{1}, B_{2}$ be Banach spaces and $u:[a, b] \rightarrow B_{1}$ be Bochner integrable. If $\Gamma: B_{1} \rightarrow B_{2}$ is a bounded linear operator then $\Gamma u$ : $[a, b] \rightarrow B_{2}$ is integrable and $\int_{E} \Gamma u(t) d t=\Gamma \int_{E} u(t) d t$ for each measurable $E \subseteq[a, b]$.

An operator $T_{1}$ is a nonlinear contraction on $B$ (a Banach space) into $B$ if for all $y_{1}, y_{2} \in B$ we have

$$
\left\|T_{1}\left(y_{1}\right)-T_{1}\left(y_{2}\right)\right\| \leq \phi\left(\left\|y_{1}-y_{2}\right\|\right)
$$

where $\phi$ is a real-valued continuous function satisfying $\phi(x)<x$ for $x>0$.

Theorem 1.8 (Krasnosel'skiı-Nashed-Wong) [16]. Let $C \subseteq B$ ( $a$ Banach space) be a closed convex subset and $T_{1}, T_{2}$ be operators on $B$ with $T_{1}(x)+$ $T_{2}(y) \in C$ for all $x, y \in C$. Suppose that

(i) $T_{2}: B \rightarrow B$ is continuous and compact $\left(T_{2}(B)\right.$ is relatively compact),

(ii) $T_{1}: B \rightarrow B$ is a nonlinear contraction.

Then there exists $y \in C$ with $T_{1}(y)+T_{2}(y)=y$.

Remark. If $T_{2}=0$ in Theorem 1.8 then in fact there exists a unique (cf. [1]) $y \in C$ with $T_{1}(y)=y$.

Theorem 1.9 (Arzelà-Ascoli) [15]. Let $B$ be a Banach space. A subset $M$ of $C([a, b], B)$ is relatively compact iff $M$ is bounded, equicontinuous and the set $\{u(t): u \in M\}$ is relatively compact in $B$ for each $t \in[a, b]$.

2. Solutions in $L^{\alpha}, \alpha>1$. Throughout this section $B$ will be a reflexive Banach space. We begin by first examining the Hammerstein integral equation

$$
y(t)=h(t)+\int_{0}^{1} k(t, s) f(s, y(s)) d s \quad \text { a.e. on }[0,1] .
$$

Theorem 2.1. Suppose $1<\alpha<\infty$ and $\beta$ is the conjugate of $\alpha$. Let $f:[0,1] \times B \rightarrow B$ and $F u(t)=f(t, u(t))$. Assume that

(2.2) $h \in L^{\alpha}([0,1], B)$,

$(2.3) \quad k:[0,1] \times[0,1] \rightarrow \mathbb{R}$ with $(t, s) \rightarrow k(t, s)$ measurable and $\int_{0}^{1} \int_{0}^{1}|k(t, s)|^{\alpha} d s d t<\infty$

(2.4) $\quad F: L^{\alpha}([0,1], B) \rightarrow L^{\beta}([0,1], B)$ is weakly continuous,

(2.5) there exists a nondecreasing continuous function $\psi:[0, \infty) \rightarrow[0, \infty)$ with $\int_{0}^{1}\|f(s, u(s))\|^{\beta} d s \leq \psi\left(\int_{0}^{1}\|u(s)\|^{\alpha} d s\right)$ for any $u \in L^{\alpha}([0,1], B)$, 


$$
2^{\alpha-1}\left(\int_{0}^{1} \int_{0}^{1}|k(t, s)|^{\alpha} d s d t\right) \limsup _{x \rightarrow \infty} \frac{\psi^{\alpha / \beta}(x)}{x}<1 .
$$

Then (2.1) has a solution $y \in L^{\alpha}([0,1], B)$.

Remark. As an example of how to apply Theorem 2.1 let $\alpha=\beta=2$, and let $0 \neq b_{0} \in B$ be fixed. Also suppose $f(t, u)=b_{0}+u$ and

$$
2\left(\int_{0}^{1} \int_{0}^{1}|k(t, s)|^{\alpha} d s d t\right)<1 .
$$

Now (2.5) is satisfied with $\psi(x)=\left\|b_{0}\right\|^{2}+2\left\|b_{0}\right\| \sqrt{x}+x$ since

$$
\begin{aligned}
\int_{0}^{1}\|f(s, u(s))\|^{2} d s & \leq \int_{0}^{1}\left(\left\|b_{0}\right\|^{2}+2\left\|b_{0}\right\|\|u(s)\|+\|u(s)\|^{2}\right) d s \\
& \leq\left\|b_{0}\right\|^{2}+2\left\|b_{0}\right\|\left(\int_{0}^{1}\|u(s)\|^{2} d s\right)^{1 / 2}+\int_{0}^{1}\|u(s)\|^{2} d s \\
& =\psi\left(\int_{0}^{1}\|u(s)\|^{2} d s\right) \quad \text { for any } u \in L^{2}([0,1], B) .
\end{aligned}
$$

In addition, (2.4) is true since if $y_{n} \rightarrow y$ in $L^{2}([0,1], B)$ then $f\left(t, y_{n}\right)=$ $b_{0}+y_{n} \rightarrow b_{0}+y=f(t, y)$ in $L^{2}([0,1], B)$. Here $\rightarrow$ denotes weak convergence. Finally, (2.6) is satisfied with the above $\psi$ and so (2.1) has a solution in $L^{2}([0,1], B)$.

Proof of Theorem 2.1. Consider the set $S$ of real numbers $x \geq 0$ which satisfy the inequality

$$
x \leq 2^{\alpha-1} \int_{0}^{1}\|h(t)\|^{\alpha} d t+2^{\alpha-1}\left(\int_{0}^{1} \int_{0}^{1}|k(t, s)|^{\alpha} d s d t\right) \psi^{\alpha / \beta}(x) .
$$

Then $S$ is bounded above, i.e. there exists a constant $M_{1}$ with

$$
x \leq M_{1} \quad \text { for all } x \in S .
$$

If (2.7) were not true then there would exist a sequence $0 \neq x_{n} \in S$ with $x_{n} \rightarrow \infty$ as $n \rightarrow \infty$ and

$$
1 \leq \frac{2^{\alpha-1} \int_{0}^{1}\|h(t)\|^{\alpha} d t}{x_{n}}+2^{\alpha-1}\left(\int_{0}^{1} \int_{0}^{1}|k(t, s)|^{\alpha} d s d t\right) \frac{\psi^{\alpha / \beta}\left(x_{n}\right)}{x_{n}} .
$$

Thus

$$
1 \leq 2^{\alpha-1}\left(\int_{0}^{1} \int_{0}^{1}|k(t, s)|^{\alpha} d s d t\right) \limsup _{x_{n} \rightarrow \infty} \frac{\psi^{\alpha / \beta}\left(x_{n}\right)}{x_{n}}
$$


which contradicts (2.6). Thus (2.7) is true. Choose $M_{0}>M_{1}$. Then

$$
2^{\alpha-1} \int_{0}^{1}\|h(t)\|^{\alpha} d t+2^{\alpha-1}\left(\int_{0}^{1} \int_{0}^{1}|k(t, s)|^{\alpha} d s d t\right) \psi^{\alpha / \beta}\left(M_{0}\right)<M_{0}
$$

for otherwise $M_{0} \in S$ and this would contradict (2.7).

Our strategy will be to apply the Schauder-Tikhonov theorem to $L^{\alpha}([0,1], B)$ endowed with the weak topology. Let

$$
K=\left\{y \in L^{\alpha}([0,1], B): \int_{0}^{1}\|y(s)\|^{\alpha} d s \leq M_{0}\right\} .
$$

Now $K$ is convex and norm closed. Hence $K$ is weakly closed by Theorem 1.5. A solution to (2.1) will be a fixed point of the operator $N: L^{\alpha}([0,1], B) \rightarrow$ $L^{\alpha}([0,1], B)$ defined by

$$
N y(t)=h(t)+\int_{0}^{1} k(t, s) f(s, y(s)) d s .
$$

We claim that $N: K \rightarrow K$ is weakly continuous and $N(K)$ is relatively weakly compact in $L^{\alpha}([0,1], B)$. If this is true then the Schauder-Tikhonov theorem (Theorem 1.6) implies that $N$ has a fixed point in $K$, i.e. (2.1) has a solution $y \in L^{\alpha}([0,1], B)$.

It remains to prove the claim. First we show $N: K \rightarrow K$. To see this notice that for a.e. $t \in[0,1]$ we have

$$
\begin{aligned}
\|N y(t)\|^{\alpha} & \leq 2^{\alpha-1}\|h(t)\|^{\alpha}+2^{\alpha-1} \int_{0}^{1}|k(t, s)|^{\alpha} d s\left(\int_{0}^{1}\|f(s, y(s))\|^{\beta} d s\right)^{\alpha / \beta} \\
& \leq 2^{\alpha-1}\|h(t)\|^{\alpha}+2^{\alpha-1} \int_{0}^{1}|k(t, s)|^{\alpha} d s \psi^{\alpha / \beta}\left(\int_{0}^{1}\|y(s)\|^{\alpha} d s\right) \\
& \leq 2^{\alpha-1}\|h(t)\|^{\alpha}+2^{\alpha-1} \int_{0}^{1}|k(t, s)|^{\alpha} d s \psi^{\alpha / \beta}\left(M_{0}\right)
\end{aligned}
$$

and so

$$
\begin{aligned}
\int_{0}^{1}\|N y(t)\|^{\alpha} d t \leq & 2^{\alpha-1} \int_{0}^{1}\|h(s)\|^{\alpha} d s \\
& +2^{\alpha-1} \psi^{\alpha / \beta}\left(M_{0}\right) \int_{0}^{1} \int_{0}^{1}|k(t, s)|^{\alpha} d s d t<M_{0}
\end{aligned}
$$

from (2.8). Consequently, $N: K \rightarrow K$. Next we show $N(K)$ is relatively weakly compact in $L^{\alpha}([0,1], B)$. Clearly, since $N(K) \subseteq K$, we see that $N(K)$ 
is bounded in $L^{\alpha}([0,1], B)$. Notice as well that

$$
N(K)(A)=\left\{\int_{A} g d t: g \in N(K)\right\}
$$

is relatively weakly compact in $B$ for every subset $A$ of $[0,1]$. This follows immediately from Theorem 1.4 and

$$
\|(N y)(A)\| \leq \int_{0}^{1}\|N y(t)\| d t \leq\left(\int_{0}^{1}\|N y(t)\|^{\alpha} d t\right)^{1 / \alpha} \leq M_{0}^{1 / \alpha} ;
$$

here $y \in K$ and $A$ is any measurable subset of $[0,1]$. Thus $N(K)(A)$ is relatively weakly compact in $B$. This, together with Theorem 1.2 (due to Brooks and Dinculeanu), implies that $N(K)$ is relatively weakly compact in $L^{\alpha}([0,1], B)$. Finally, it remains to show that $N: L^{\alpha}([0,1], B) \rightarrow$ $L^{\alpha}([0,1], B)$ is weakly continuous, i.e.

$$
\text { if } y_{n} \rightarrow y \text { in } L^{\alpha}([0,1], B) \text { then } N y_{n} \rightarrow N y \text { in } L^{\alpha}([0,1], B) \text {; }
$$

hence $\left(y_{n}\right)$ is a net in $L^{\alpha}([0,1], B)$. Let $\phi \in\left(L^{\alpha}([0,1], B)^{*}\right.$. Then there exists $g \in L^{\beta}\left([0,1], B^{*}\right)$ with (see Theorem 1.3)

$$
\phi\left(N y_{n}-N y\right)=\int_{0}^{1} g(t)\left(\int_{0}^{1} k(t, s)\left[f\left(s, y_{n}(s)\right)-f(s, y(s))\right] d s\right) d t .
$$

Theorem 1.7 and changing the order of integration yield

$$
\begin{aligned}
\phi\left(N y_{n}-N y\right) & =\int_{0}^{1} \int_{0}^{1} k(t, s) g(t)\left(f\left(s, y_{n}(s)\right)-f(s, y(s))\right) d s d t \\
& =\int_{0}^{1} \int_{0}^{1} k(t, s) g(t)\left(f\left(s, y_{n}(s)\right)-f(s, y(s))\right) d t d s \\
& =\int_{0}^{1}\left(\int_{0}^{1} k(t, s) g(t) d t\right)\left(f\left(s, y_{n}(s)\right)-f(s, y(s))\right) d s \\
& =\int_{0}^{1} g_{1}(s)\left(f\left(s, y_{n}(s)\right)-f(s, y(s))\right) d s
\end{aligned}
$$

where $g_{1}(s)=\int_{0}^{1} k(t, s) g(t) d t$. This, together with (2.4) and $g_{1} \in$ $L^{\alpha}\left([0,1], B^{*}\right)$ (note $(2.6)$ and $g \in L^{\beta}\left([0,1], B^{*}\right)$ ), implies that $N: L^{\alpha}([0,1], B)$ $\rightarrow L^{\alpha}([0,1], B)$ is weakly continuous.

The Schauder-Tikhonov theorem guarantees that $N$ has a fixed point in $K$. 
Essentially the same reasoning as in Theorem 2.1 immediately establishes an existence result for the Volterra integral equation

$$
y(t)=h(t)+\int_{0}^{t} k(t, s) f(s, y(s)) d s \quad \text { a.e. on }[0, T] .
$$

Theorem 2.2. Suppose $1<\alpha<\infty$ and $\beta$ is the conjugate of $\alpha$. Let $f$ : $[0, T] \times B \rightarrow B$ where $B$ is a reflexive Banach space and $F u(t)=f(t, u(t))$. Assume that

(2.10) $h \in L^{\alpha}([0, T], B)$,

$(2.11) \quad k:[0, T] \times[0, T] \rightarrow \mathbb{R}$ with $(t, s) \rightarrow k(t, s)$ measurable and $\int_{0}^{T} \int_{0}^{t}|k(t, s)|^{\alpha} d s d t<\infty$

(2.12) $F: L^{\alpha}([0, T], B) \rightarrow L^{\beta}([0, T], B)$ is weakly continuous,

(2.13) there exists a nondecreasing continuous function $\psi:[0, \infty) \rightarrow$ $[0, \infty)$ with $\int_{0}^{t}\|f(s, u(s))\|^{\beta} d s \leq \psi\left(\int_{0}^{t}\|u(s)\|^{\alpha} d s\right)$ for $t \in[0, T]$ and any $u \in L^{\alpha}([0, T], B)$,

$$
2^{\alpha-1}\left(\int_{0}^{T} \int_{0}^{t}|k(t, s)|^{\alpha} d s d t\right) \limsup _{x \rightarrow \infty} \frac{\psi^{\alpha / \beta}(x)}{x}<1
$$

Then (2.9) has a solution $y \in L^{\alpha}([0, T], B)$.

However, it is possible to improve this result.

TheOREM 2.3. Let $1<\alpha<\infty$ and $\beta$ be the conjugate of $\alpha$. Suppose $f:[0, T] \times B \rightarrow B$ and $F u(t)=f(t, u(t))$. Assume that (2.10)-(2.13) hold. In addition, assume that

$$
2^{\alpha-1}\left(\int_{0}^{T}\|h(s)\|^{\alpha} d s+\int_{0}^{T} \int_{0}^{t}|k(t, s)|^{\alpha} d s d t\right)<\int_{0}^{\infty} \frac{d u}{1+\psi^{\alpha / \beta}(u)}
$$

Then (2.9) has a solution $y \in L^{\alpha}([0, T], B)$.

Proof. Let

$$
I(z)=\int_{0}^{z} \frac{d u}{1+\psi^{\alpha / \beta}(u)}
$$

and

$$
a(t)=I^{-1}\left(2^{\alpha-1} \int_{0}^{t}\|h(s)\|^{\alpha} d s+2^{\alpha-1} \int_{0}^{t} \int_{0}^{s}|k(s, x)|^{\alpha} d x d s\right) .
$$

Now let

$$
K=\left\{y \in L^{\alpha}([0, T], B): \int_{0}^{t}\|y(s)\|^{\alpha} d s \leq a(t)\right\}
$$


The set $K$ is convex and weakly closed. Also, a solution to (2.9) will be a fixed point of the operator $N: L^{\alpha}([0, T], B) \rightarrow L^{\alpha}([0, T], B)$ defined by

$$
N y(s)=h(s)+\int_{0}^{s} k(s, x) f(x, y(x)) d x .
$$

We claim that $N: K \rightarrow K$. To see this notice for a.e. $s \in[0, T]$ that

$$
\begin{aligned}
\|N y(s)\|^{\alpha} & \leq 2^{\alpha-1}\|h(s)\|^{\alpha}+2^{\alpha-1} \int_{0}^{s}|k(s, x)|^{\alpha} d x\left(\int_{0}^{s}\|f(x, y(x))\|^{\beta} d x\right)^{\alpha / \beta} \\
& \leq 2^{\alpha-1}\|h(s)\|^{\alpha}+2^{\alpha-1} \int_{0}^{s}|k(s, x)|^{\alpha} d x \psi^{\alpha / \beta}\left(\int_{0}^{s}\|y(x)\|^{\alpha} d x\right) \\
& \leq\left(2^{\alpha-1}\|h(s)\|^{\alpha}+2^{\alpha-1} \int_{0}^{s}|k(s, x)|^{\alpha} d x\right)\left(1+\psi^{\alpha / \beta}(a(s))\right) .
\end{aligned}
$$

Thus for $t \in[0, T]$ we have

$$
\begin{aligned}
\int_{0}^{t} \| N y(s) & \|^{\alpha} d s \\
& \leq \int_{0}^{t}\left(2^{\alpha-1}\|h(s)\|^{\alpha}+2^{\alpha-1} \int_{0}^{s}|k(s, x)|^{\alpha} d x\right)\left(1+\psi^{\alpha / \beta}(a(s))\right) d s \\
& =\int_{0}^{t} a^{\prime}(s) d s=a(t)
\end{aligned}
$$

since (2.16) implies

$$
\int_{0}^{a(s)} \frac{d u}{1+\psi^{\alpha / \beta}(u)}=2^{\alpha-1}\left(\int_{0}^{s}\|h(x)\|^{\alpha} d x+\int_{0}^{s} \int_{0}^{z}|k(z, x)|^{\alpha} d x d z\right) .
$$

Consequently, $N y \in K$ and so $N: K \rightarrow K$. Essentially the same reasoning as in Theorem 2.1 shows that $N(K)$ is relatively weakly compact in $L^{\alpha}([0, T], B)$ and $N: K \rightarrow K$ is weakly continuous. The Schauder-Tikhonov theorem now guarantees a fixed point of $N$ in $K$.

3. Solutions in $C$. Throughout this section, $B$ will be a real Banach space. We consider first the Volterra integral equation

$$
y(t)=h(t)+\int_{0}^{t} k(t, s) f(s, y(s)) d s, \quad t \in[0, T] .
$$

We will assume that $f:[0, T] \times B \rightarrow B$ is a $L^{\beta}$-Carathéodory function; here $\beta \geq 1$. By this we mean that 
(i) the map $t \rightarrow f(t, z)$ is measurable (Bochner) for all $z \in B$,

(ii) the map $z \rightarrow f(t, z)$ is continuous for almost all $t \in[0, T]$,

(iii) for each $r>0$ there exists $\mu_{r} \in L^{\beta}([0, T], \mathbb{R})$ such that $\|z\| \leq r$ implies $\|f(t, z)\| \leq \mu_{r}(t)$ for almost all $t \in[0, T]$.

Theorem 3.1. Let $1 \leq \alpha \leq \infty$ and $\beta$ be the conjugate of $\alpha$. Suppose $f:[0, T] \times B \rightarrow B$ has the decomposition $f=f_{1}+f_{2}$ where $f_{1}$ and $f_{2}$ are $L^{\beta}$-Carathéodory functions. Assume that

(3.2) $h \in C([0, T], B)$,

(3.3) $k(t, s) \in L^{\alpha}([0, T], \mathbb{R})$ for each $t \in[0, T]$ and the map $t \rightarrow k(t, s)$ is continuous from $[0, T]$ to $L^{\alpha}([0, T], \mathbb{R})$,

(3.4) there exists a nondecreasing continuous function $\Phi:[0, \infty) \rightarrow[0, \infty)$ with $\int_{0}^{t}\|k(t, s) f(s, u(s))\| d s \leq \Phi\left(\int_{0}^{t}\|u(s)\| d s\right)$ for $t \in[0, T]$ and any $u \in C([0, T], B)$,

$T<\int_{0}^{\infty} \frac{d u}{\Phi(u)+h_{0}}$ where $h_{0}=\sup _{[0, T]}\|h(t)\|$.

Let

$$
J(z)=\int_{0}^{z} \frac{d u}{\Phi(u)+h_{0}}
$$

and notice that $J:[0, \infty) \rightarrow[0, \infty)$ is strictly increasing. Define

$$
M_{1}=J^{-1}(T) \quad \text { and } \quad M_{0}=h_{0}+\Phi\left(M_{1}\right) .
$$

In addition, suppose that

(3.7) for each $t \in[0, T]$ the set $\left\{\int_{0}^{t} k(t, s) f_{2}(s, u(s)) d s: u \in C([0, T], B)\right.$ with $\|u(s)\| \leq M_{0}$ for all $\left.s \in[0, T]\right\}$ is relatively compact,

and

(3.8) there exists a continuous $Q:[0, T] \rightarrow[0, \infty)$ such that

$$
\begin{aligned}
\sup _{[0, T]} \| e^{-Q(t)} \int_{0}^{t} k(t, s)\left[f_{1}(s, u(s))-\right. & \left.f_{1}(s, v(s))\right] d s \| \\
& \leq \phi\left(\frac{1}{2} \sup _{[0, T]} e^{-Q(t)}\|u(t)-v(t)\|\right)
\end{aligned}
$$

for all $u, v \in C([0, T], B)$ with $\|u(s)\|,\|v(s)\| \leq M_{0}$ for all $s \in[0, T]$; here $\phi$ is a real-valued nondecreasing continuous function satisfying $\phi(x)<x$ for $x>0$.

Then (3.1) has a solution $y \in C([0, T], B)$.

Remarks. (i) Let $k \equiv 1$ and suppose there exists $q \in L^{1}([0, T], \mathbb{R})$ with

$$
\left\|f_{1}(t, u)-f_{1}(t, v)\right\| \leq q(t)\|u-v\|
$$


for a.e. $t \in[0, T]$ and all $u, v \in B$ with $\|u\| \leq M_{0},\|v\| \leq M_{0}$. Then (3.8) is satisfied. To see this consider any $u, v \in C([0, T], B)$ with $\|u(s)\|,\|v(s)\| \leq$ $M_{0}$ for $s \in[0, T]$. With $Q(t)=2 \int_{0}^{t} q(s) d s$ we have

$$
\begin{aligned}
\sup _{[0, T]}\left\|e^{-Q(t)} \int_{0}^{t}\left[f_{1}(s, u(s))-f_{1}(s, v(s))\right] d s\right\| \\
\leq \sup _{t \in[0, T]} e^{-Q(t)} \int_{0}^{t} e^{Q(s)} q(s) e^{-Q(s)}\|u(s)-v(s)\| d s \\
\leq\|u-v\|_{Q} \sup _{t \in[0, T]} e^{-Q(t)} \frac{1}{2}\left[e^{Q(t)}-1\right] \\
=\frac{1}{2}\left(1-e^{-Q(T)}\right)\|u-v\|_{Q}
\end{aligned}
$$

where $\|u-v\|_{Q}=\sup _{[0, T]} e^{-Q(t)}\|u(t)-v(t)\|$. Clearly (3.8) is satisfied with $\phi(x)=\left(1-e^{-Q(T)}\right) x$.

(ii) We can replace $\frac{1}{2}$ in (3.8) by 1 if $B=H$, a Hilbert space.

(iii) We can replace $e^{-Q(t)}$ in (3.8) with an arbitrary weight function $w(t)$.

(iv) If $f_{2}=0$ in Theorem 3.1 then in fact (3.1) has a unique solution $y \in C([0, T], B)$.

Proof of Theorem 3.1. Consider the modified Volterra equation (3.9) $y(t)=h(t)+\int_{0}^{t} k(t, s)\left[f_{1}(s, r(y(s)))+f_{2}(s, r(y(s)))\right] d s, \quad t \in[0, T]$,

where $r: B \rightarrow \overline{B\left(0, M_{0}\right)}=\left\{y:\|y\| \leq M_{0}\right\}$ defined by

$$
r(u)= \begin{cases}u, & \|u\| \leq M_{0}, \\ M_{0} u /\|u\|, & \|u\|>M_{0},\end{cases}
$$

is the radial retraction; $M_{0}$ is as described in (3.6). Recall the radial retraction $r$ is Lipschitz $[8,12]$ and in fact

$$
\left\|r\left(u_{1}\right)-r\left(u_{2}\right)\right\| \leq 2\left\|u_{1}-u_{2}\right\| \quad \text { for all } u_{1}, u_{2} \in B .
$$

R e m ark. If $B=H$, a real Hilbert space, then in fact $r$ is nonexpansive $[10,12]$.

Let us endow $C([0, T], B)$ with the norm

$$
\|u\|_{Q}=\sup _{t \in[0, T]} e^{-Q(t)}\|u(t)\| .
$$


A solution to (3.9) is a fixed point of the operator $S: C([0, T], B) \rightarrow$ $C([0, T], B)$ defined by

$$
S y(t)=h(t)+\int_{0}^{t} k(t, s) f(s, r(y(s))) d s \equiv\left(T_{1} y\right)(t)+\left(T_{2} y\right)(t)
$$

where

$$
\begin{aligned}
& \left(T_{1} y\right)(t)=h(t)+\int_{0}^{t} k(t, s) f_{1}(s, r(y(s))) d s, \\
& \left(T_{2} y\right)(t)=\int_{0}^{t} k(t, s) f_{2}(s, r(y(s))) d s .
\end{aligned}
$$

Now $T_{1}: C([0, T], B) \rightarrow C([0, T], B)$ is a nonlinear contraction since for $u, v \in C([0, T], B)$ we have, with $\|\cdot\|_{Q}$ as described in (3.11),

$$
\begin{aligned}
\left\|T_{1}(u)-T_{1}(v)\right\|_{Q} & =\sup _{[0, T]}\left\|e^{-Q(t)} \int_{0}^{t} k(t, s)\left[f_{1}(s, r(u(s)))-f_{1}(s, r(v(s)))\right] d s\right\| \\
& \leq \phi\left(\frac{1}{2} \sup _{[0, T]} e^{-Q(t)}\|r(u(t))-r(v(t))\|\right) \\
& \leq \phi\left(\sup _{[0, T]} e^{-Q(t)}\|u(t)-v(t)\|\right)=\phi\left(\|u-v\|_{Q}\right),
\end{aligned}
$$

using (3.8), (3.10) and the fact that $\phi$ is nondecreasing.

Next we show that $T_{2}: C([0, T], B) \rightarrow C([0, T], B)$ is continuous and compact. To see continuity let $y_{n} \rightarrow y$ in $C([0, T], B)$. Now $\left\|r\left(y_{n}(s)\right)\right\| \leq M_{0}$ and $\|r(y(s))\| \leq M_{0}$ for all $s \in[0, T]$. Also, there exists $\mu \in L^{\beta}([0, T], \mathbb{R})$ with $\left\|f_{2}(t, u)\right\| \leq \mu(t)$ for a.e. $t \in[0, T]$ and all $\|u\| \leq M_{0}$. In addition, for each $t \in[0, T]$ we have

$$
k(t, s) f_{2}\left(s, r\left(y_{n}(s)\right)\right) \rightarrow k(t, s) f_{2}(s, r(y(s))) \quad \text { for a.e. } s \in[0, T]
$$

and this, together with the Lebesgue dominated convergence theorem, implies $T_{2} y_{n}(s) \rightarrow T_{2} y(s)$ pointwise on $[0, T]$. Next we show the convergence is uniform and this of course implies $T_{2}: C([0, T], B) \rightarrow C([0, T], B)$ is continuous. Let $t, t_{1} \in[0, T]$ with $t_{1}<t$. Then

$$
\begin{aligned}
& \left\|T_{2} y_{n}(t)-T_{2} y_{n}\left(t_{1}\right)\right\| \\
& \leq\left\|h(t)-h\left(t_{1}\right)\right\|+\int_{0}^{t_{1}}\left|k(t, s)-k\left(t_{1}, s\right)\right|\left\|f\left(s, r\left(y_{n}(s)\right)\right)\right\| d s \\
& \quad+\int_{t_{1}}^{t}|k(t, s)|\left\|f\left(s, r\left(y_{n}(s)\right)\right)\right\| d s
\end{aligned}
$$




$$
\begin{aligned}
\leq & \left\|h(t)-h\left(t_{1}\right)\right\|+\left(\int_{0}^{T}\left|k(t, s)-k\left(t_{1}, s\right)\right|^{\alpha} d s\right)^{1 / \alpha}\left(\int_{0}^{T} \mu^{\beta}(s) d s\right)^{1 / \beta} \\
& +\sup _{t \in[0, T]}\left(\int_{0}^{T}|k(t, s)|^{\alpha} d s\right)^{1 / \alpha}\left(\int_{t_{1}}^{t} \mu^{\beta}(s) d s\right)^{1 / \beta} .
\end{aligned}
$$

A similar bound can be obtained for $\left\|T_{2} y(t)-T_{2} y\left(t_{1}\right)\right\|$. Thus for any $\varepsilon>0$ there exists $\delta>0$ such that $t, t_{1} \in[0, T]$ and $\left|t-t_{1}\right|<\delta$ imply

(3.12) $\left\|T_{2} y_{n}(t)-T_{2} y_{n}\left(t_{1}\right)\right\|<\varepsilon$ for all $n$ and $\left\|T_{2} y(t)-T_{2} y\left(t_{1}\right)\right\|<\varepsilon$.

Now (3.12), together with the fact that $T_{2} y_{n}(s) \rightarrow T_{2} y(s)$ pointwise on $[0, T]$, implies that the convergence is uniform. Consequently, $T_{2}: C([0, T], B) \rightarrow$ $C([0, T], B)$ is continuous. In addition, the Arzelà-Ascoli theorem (Theorem 1.9), together with (3.7) and the ideas used to prove (3.12), implies that $T_{2}: C([0, T], B) \rightarrow C([0, T], B)$ is compact.

The Krasnosel'skiu-Nashed-Wong fixed point theorem guarantees a fixed point of $S$, i.e. (3.9) has a solution $y \in C([0, T], B)$. We now show that $y$ is a solution of (3.1).

Remark. It is worth remarking here that (3.4) and (3.5) are only needed, so far, to define $M_{0}$; in fact, we have shown that (3.9) has a solution for any constant $M_{0}$.

Now for each $t \in(0, T)$,

$$
\begin{aligned}
\|y(t)\| & \leq\|h(t)\|+\int_{0}^{t}|k(t, s)|\|f(s, r(y(s)))\| d s \\
& \leq\|h(t)\|+\Phi\left(\int_{0}^{t}\|r(y(x))\| d x\right) \leq h_{0}+\Phi\left(\int_{0}^{t}\|y(x)\| d x\right),
\end{aligned}
$$

using (3.4) and the fact that $\|r(y(x))\| \leq\|y(x)\|, x \in[0, T]$; here $h_{0}=$ $\sup _{[0, T]}\|h(t)\|$. Consequently, integration from 0 to $t$ yields

so

$$
\int_{0}^{t}\|y(x)\| d x \frac{d u}{\Phi(u)+h_{0}} \leq t \leq T
$$

$$
\int_{0}^{t}\|y(x)\| d x \leq J^{-1}(T)=M_{1} \quad \text { for } t \in[0, T] .
$$

Also, we have

$$
\|y(t)\| \leq h_{0}+\Phi\left(\int_{0}^{t}\|y(x)\| d x\right) \leq h_{0}+\Phi\left(M_{1}\right)=M_{0} .
$$

Thus $f(s, r(y(s)))=f(s, y(s))$, so $y$ is a solution of $(3.1)$. 
R e m a r k. $\Phi\left(\int_{0}^{t}\|y(x)\| d x\right)$ in (3.4) could be replaced by $\Phi\left(\int_{0}^{t}\|y(x)\|^{\sigma} d x\right)$ for some constant $\sigma \geq 1$ and existence of a solution to (3.1) is again guaranteed (of course (3.5) has to be appropriately adjusted).

Next we examine the Hammerstein integral equation

$$
y(t)=h(t)+\int_{0}^{1} k(t, s) f(s, y(s)) d s, \quad t \in[0,1] .
$$

Throughout, $f:[0,1] \times B \rightarrow B$ will be a $L^{\beta}$-Carathéodory function. Also, the following will be satisfied (here $1 \leq \alpha \leq \infty$ and $\beta$ is the conjugate to $\alpha$ ):

(3.14) $h \in C([0,1], B)$,

(3.15) $k(t, s) \in L^{\alpha}([0,1], \mathbb{R})$ for each $t \in[0,1]$ and the map $t \rightarrow k(t, s)$ is continuous from $[0,1]$ to $L^{\alpha}([0,1], \mathbb{R})$,

(3.16) there exists a nondecreasing continuous function $\theta:[0, \infty) \rightarrow$ $[0, \infty)$ with $\int_{0}^{1}\|f(s, u(s))\|^{\beta} d s \leq \theta\left(\int_{0}^{1}\|u(s)\|^{\alpha} d s\right)$ for any $u \in$ $C([0,1], B)$,

$$
2^{\alpha-1}\left(\int_{0}^{1} \int_{0}^{1}|k(t, s)|^{\alpha} d s d t\right) \limsup _{x \rightarrow \infty} \frac{\theta^{\alpha / \beta}(x)}{x}<1 .
$$

Remark. (3.17) has an obvious analogue when $\alpha=\infty$.

Consider the set $S$ of real numbers $x \geq 0$ which satisfy the inequality

$$
x \leq 2^{\alpha-1} \int_{0}^{1}\|h(t)\|^{\alpha} d t+2^{\alpha-1}\left(\int_{0}^{1} \int_{0}^{1}|k(t, s)|^{\alpha} d s d t\right) \theta^{\alpha / \beta}(x) .
$$

Then $S$ is bounded above (see Theorem 2.1), i.e. there exists a constant $M_{2}$ with

$$
x \leq M_{2} \quad \text { for all } x \in S .
$$

TheOREM 3.2. Suppose $f:[0,1] \times B \rightarrow B$ has the decomposition $f=$ $f_{1}+f_{2}$ where $f_{1}$ and $f_{2}$ are $L^{\beta}$-Carathéodory functions. Assume that (3.14)(3.17) hold. Let $M_{2}$ be as in (3.18) and define

$$
M_{3}=\sup _{[0,1]}\|h(t)\|+\sup _{[0,1]}\left(\int_{0}^{1}|k(t, s)|^{\alpha} d s\right)^{1 / \alpha} \theta^{1 / \beta}\left(M_{2}\right) .
$$

In addition, assume that

$$
\begin{aligned}
& \text { for each } t \in[0,1] \text { the set }\left\{\int_{0}^{1} k(t, s) f_{2}(s, u(s)) d s: u \in C([0,1], B)\right. \\
& \text { with } \left.\|u(s)\| \leq M_{3} \text { for all } s \in[0,1]\right\} \text { is relatively compact, } \\
& \text { there exists a continuous } Q:[0,1] \rightarrow[0, \infty) \text { such that }
\end{aligned}
$$




$$
\begin{aligned}
& \sup _{[0,1]} \| e^{-Q(t)} \int_{0}^{1} k(t, s)\left[f_{1}(s, u(s))-\right.\left.f_{1}(s, v(s))\right] d s \| \\
& \leq \phi\left(\frac{1}{2} \sup _{[0,1]} e^{-Q(t)}\|u(t)-v(t)\|\right)
\end{aligned}
$$

for all $u, v \in C([0,1], B)$ with $\|u(s)\|,\|v(s)\| \leq M_{3}$ for all $s \in[0,1]$; here $\phi$ is a real-valued nondecreasing continuous function satisfying $\phi(x)<x$ for $x>0$.

Then (3.13) has a solution $y \in C([0,1], B)$.

Pr o of. Consider the modified Hammerstein equation

$$
y(t)=h(t)+\int_{0}^{1} k(t, s) f(s, r(y(s))) d s, \quad t \in[0,1],
$$

where $r: B \rightarrow \overline{B\left(0, M_{3}\right)}=\left\{y:\|y\| \leq M_{3}\right\}$ is the radial retraction. Essentially the same reasoning as in Theorem 3.1 implies that (3.22) has a solution $y \in C([0,1], B)$.

Now for $t \in(0,1)$ we have

$$
\|y(t)\| \leq\|h(t)\|+\int_{0}^{1}|k(t, s)|\|f(s, r(y(s)))\| d s .
$$

We will just consider the case $1 \leq \alpha<\infty$. The case $\alpha=\infty$ is similar. Hölder's inequality, together with (3.16), yields

$$
\begin{aligned}
\int_{0}^{1}\|y(t)\|^{\alpha} d t \leq & 2^{\alpha-1} \int_{0}^{1}\|h(t)\|^{\alpha} d t \\
& +2^{\alpha-1}\left(\int_{0}^{1} \int_{0}^{1}|k(t, s)|^{\alpha} d s d t\right) \theta^{\alpha / \beta}\left(\int_{0}^{1}\|r(y(s))\|^{\alpha} d s\right) \\
\leq & 2^{\alpha-1} \int_{0}^{1}\|h(t)\|^{\alpha} d t \\
& +2^{\alpha-1}\left(\int_{0}^{1} \int_{0}^{1}|k(t, s)|^{\alpha} d s d t\right) \theta^{\alpha / \beta}\left(\int_{0}^{1}\|y(s)\|^{\alpha} d s\right)
\end{aligned}
$$

since $\theta$ is nondecreasing and $\|r(y(s))\| \leq\|y(s)\|, s \in[0,1]$. This, together 
with (3.18), yields

$$
\int_{0}^{1}\|y(s)\|^{\alpha} d s \leq M_{2} .
$$

Returning to (3.23), for $t \in[0,1]$ we have

$$
\begin{aligned}
\|y(t)\| & \leq \sup _{[0,1]}\|h(t)\|+\left(\int_{0}^{1}|k(t, s)|^{\alpha} d s\right)^{1 / \alpha} \theta^{1 / \beta}\left(\int_{0}^{1}\|r(y(s))\|^{\alpha} d s\right) \\
& \leq \sup _{[0,1]}\|h(t)\|+\sup _{[0,1]}\left(\int_{0}^{1}|k(t, s)|^{\alpha} d s\right)^{1 / \alpha} \theta^{1 / \beta}\left(M_{2}\right)=M_{3}
\end{aligned}
$$

since $\int_{0}^{1}\|r(y(s))\|^{\alpha} d s \leq \int_{0}^{1}\|y(s)\|^{\alpha} d s \leq M_{2}$. Since $\|y(t)\| \leq M_{3}$ for $t \in$ $[0,1]$, we find that $f(s, r(y(s)))=f(s, y(s))$ and the result follows.

\section{References}

[1] D. Boyd and J. S. W. Wong, On nonlinear contractions, Proc. Amer. Math. Soc. 20 (1969), 458-464.

[2] J. K. Brooks and N. Dinculeanu, Weak compactness in spaces of Bochner integrable functions and applications, Adv. in Math. 24 (1977), 172-188.

[3] C. Corduneanu, Integral Equations and Stability of Feedback Systems, Academic Press, New York, 1973.

[4] -, Integral Equations and Applications, Cambridge Univ. Press, New York, 1990.

[5] - , Perturbations of linear abstract Volterra equations, J. Integral Equations Appl. 2 (1990), 393-401.

[6] -, Abstract Volterra equations and weak topologies, in: Delay Differential Equations and Dynamical Systems, S. Busenberg and M. Martelli (eds.), Lecture Notes in Math. 1475, Springer, 110-116.

[7] J. B. Conway, A Course in Functional Analysis, Springer, Berlin, 1990.

[8] D. G. DeFigueiredo and L. A. Karlovitz, On the radial projection in normed spaces, Bull. Amer. Math. Soc. 73 (1967), 364-368.

[9] J. Diestel and J. J. Uhl, Jr., Vector Measures, Math. Surveys 15, Amer. Math. Soc., Providence, 1977.

[10] J. Dugundji and A. Granas, Fixed Point Theory, Monograf. Mat. 61, PWN, Warszawa, 1982.

[11] N. D unford and J. T. Schwartz, Linear Operators, Interscience Publ. Inc., Wiley, New York, 1958.

[12] C. F. Dunkl and K. S. Williams, A simple norm inequality, Amer. Math. Monthly 71 (1964), 53-54.

[13] G. Gripenberg, S. O. Londen and O. Staffans, Volterra Integral and Functional Equations, Cambridge Univ. Press, New York, 1990.

[14] R. B. Guenther and J. W. Lee, Some existence results for nonlinear integral equations via topological transversality, J. Integral Equations Appl. 5 (1993), 195209.

[15] R. H. Martin, Jr., Nonlinear Operators and Differential Equations in Banach Spaces, Wiley, New York, 1976. 
[16] M. Z. Nashed and J. S. W. Wong, Some variants of a fixed point theorem of Krasnosel'skiu and applications to nonlinear integral equations, J. Math. Mech. 18 (1969), 767-777.

[17] K. Yosida, Functional Analysis, Springer, Berlin, 1971.

DEPARTMENT OF MATHEMATICS

UNIVERSITY COLLEGE GALWAY

GALWAY, IRELAND

Reçu par la Rédaction le 18.5.1994

Révisé le 30.6.1994 